Ann. Biol. anim. Bioch. Biophys., 1978, 18 (1), 133-136.

\title{
Etude de l'effet du pyrrolidonecarboxylate de calcium sur la formation des macromolécules de la matrice osseuse
}

\author{
par B. PHAN-DINH-TUY, E. MOCZAR
}

Laboratoire de Biochimie du Tissu conjonctif

Faculté de Médecine, 6, rue du Général Sarrail, 94000 Créteil.

Summary. Effect of calcium pyrrolidon carboxylate on macromolecule formation in bone matrix.

${ }^{14} \mathrm{C}$ - and ${ }^{45} \mathrm{Ca}$ labelled calcium pyrrolidon carboxylate (PCA-Ca) was given orally to rats. The incorporated radioactivity and the nature of the radioactive aminoacids were determined in various tissues. The incorporation of ${ }^{14} \mathrm{C}-\mathrm{PCA}-\mathrm{Ca}$ and its transformation products was more intense in liver and bones than in other organs. ${ }^{45} \mathrm{Ca}$ incorporation was the strongest in the bones. An important fraction of PCA was transformed in proline and hydroxyproline in the bones and skin. Incorporation studies ${ }^{14} \mathrm{C}$-proline and ${ }^{35} \mathrm{SO}_{4} \mathrm{Na}_{2}$ in fracture callus of rat indicated an activation of protein as well as of glycosaminoglycan metabolism after calcium pyrrolidon carboxylate administration.

Le sel calcique de l'acide pyrrolidonecarboxylique (APC-Ca) a été introduit récemment dans la thérapie des troubles du métabolisme de l'os. Cet anion est complètement atoxique, il exerce aussi une action protectrice sur les cellules nerveuses et hépatiques (Di Rosa, 1967). La constante de dissociation en solution ( $\mathrm{Kd}: 1,3$ 10 $^{-3}$ ) et d'association avec le calcium $\mathrm{C}=\left(\mathrm{ACa}^{+}\right) /\left(\mathrm{A}^{-}\right) *\left(\mathrm{Ca}^{2+}\right)=2,89$ de l'APC ont été comparées avec celles de quelques autres acides organiques (acétique, lactique, gluconique ef propionique) (Phan-Dinh-Tuy et Moczar, données non publiées). Le rapport $\mathrm{Kd} / \mathrm{C}$ de l'APC $\left(4010^{-5}\right)$ est environ 40 fois supérieur à celui des autres acides étudiés $\left(1-1,510^{-5}\right)$. L'APC est un acide fort, son $\mathrm{Kd}$ est environ 50 fois supérieur à celui des acides carboxyliques éfudiés.

La calcification in vitro du collagène (Bachra, 1967) est intensifiée en présence d'APC-Ca. Elle apparaît plus forte que pour les sels calciques dont l'anion est un complexant comme le lactate et le gluconate (Phan-Dinh-Tuy et Moczar, données non publiées).

Prenant en considération ces résultats, il nous a paru intéressant d'étudier certains aspects du métabolisme de l'APC et l'influence de l'administration de ce sel sur la biosynthèse des macromolécules du tissu osseux.

\footnotetext{
* Concentration en base conjuguée de l'acide (AH) (ici : APC).
} 


\section{Matériel et méthodes.}

1) Étude de la répartition de la radioactivité après administration per os d'APC-Ca radioactif.

Des rats mâles adultes Wistar pesant $300 \pm 15 \mathrm{~g}$ ont été répartis en 2 lots. Chaque rat a reçu deux doses de $12,5 \mathrm{mg}$ d'APC-Ca marqué, soit au ${ }^{14} \mathrm{C}$ (L-(U $\left.{ }^{14} \mathrm{C}\right)-\mathrm{APC}-\mathrm{Ca}$, $1,1 \mu \mathrm{Ci} / \mathrm{mg})$ pour le premier lot, soit au ${ }^{45} \mathrm{Ca}\left(\mathrm{APC}-{ }^{45} \mathrm{Ca}, I \mu \mathrm{Ci} / \mathrm{mg}\right)$ pour le deuxième lot. Le sel a été administré à 2 reprises par sonde gastrique, en solution aqueuse à $16 \mathrm{~h}$ d'intervalle. Les animaux ont été sacrifiés $5 \mathrm{~h}$ après la deuxième injection.

Au cours d'une seconde expérience de jeunes rats pesant $50 \pm 2,5 \mathrm{~g}$ ont été traités selon le même protocole en utilisant exclusivement du L-(U $\left.{ }^{14} \mathrm{C}\right)$ APC-Ca d'une activité spécifique plus élevée $(10 \mu \mathrm{Ci} / \mathrm{mg})$. Les organes (os longs : tibias-péronés, fémurs, humérus ; la calotte crânienne, le cerveau, l'aorte, le foie, la rate, les reins, un fragment de peau dorsale et abdominale) ont été hydrolysés par $6 \mathrm{~N}-\mathrm{HCl}(24 \mathrm{~h})$ ef la radioactivité a été déterminée dans un compteur à scintillation liquide. Les acides aminés ont été séparés par électrophorèse sous haute tension sur papier ou sur couche mince de cellulose dans un tampon de pyridine-acétate $\mathrm{pH}$ 3,5 (Moczar, Robert et Robert, 1972). La radioactivité des zones éluées a été déterminée par compłage en scintillation liquide. L'activité spécifique de la proline (pro) et de l'hydroxyproline (hyp) a été évaluée selon la méthode de Rojkind ef Gonzalez (1974).

2) Etude de la formation de cals osseux chez le rat.

Des rats mâles Wistar pesant $300 \pm 15 \mathrm{~g}$ ont été divisés en trois groupes $(6 \mathrm{rats}$ par groupe). Un lot témoin et deux lots qui reçoivent respectivement 100 et $300 \mathrm{mg}$ d'APC-Ca par jour après fracture bilatérale de la diaphyse tibiale sous anesthésie.

Des rats de chaque lot sont prélevés après $5,7,12,13,16$ et 23 jours de traitement. Les cals osseux sont débités en tranches de $200 \mu \mathrm{m}$ environ au microtome et incubés dans un tampon Krebs-Ringer phosphate en présence de $50 \mu \mathrm{Ci} \mathrm{Na}{ }_{2}{ }^{35} \mathrm{SO}_{4}$ ou de $10 \mu \mathrm{Ci}{ }^{14} \mathrm{C}$-proline selon Penttinen (1973). L'activité spécifique de la proline et de l'hydroxyproline et l'activité du ${ }^{35}$ S incorporé ont été déterminées respectivement selon les techniques de Rojkind et Gonzalez (1974) et une variante de la méthode de Penttinen (1973).

\section{Résultats et discussion.}

La répartition de la radioactivité dans les organes après administration soit de ${ }^{14} \mathrm{C}-\mathrm{APC}$-Ca soit de ${ }^{45} \mathrm{Ca}-\mathrm{APC}$ est rapportée dans le tableau 1 . Les résultats indiquent une incorporation préférentielle de ${ }^{45} \mathrm{Ca}$ dans l'os. L'incorporation de ${ }^{14} \mathrm{C}$ (noyau pyrrolidone) est la plus élevée dans le foie et dans les os. Ce phénomène est particulièrement marqué chez les jeunes rats $(50 \mathrm{~g})$. Lange et Carey (1966) et Ramakrishna et al. (1970) ont démontré qu'une fraction importante de l'APC est transformée en acide glutamique et en acide $\gamma$-amino butyrique après administration per os. Nos 
résultats (tabl. 1) indiquent qu'en utilisant des doses fortes d'APC-Ca et après électrophorèse des hydrolysats acides des différents organes, 85 p. 100 environ de la radioactivité incorporée se retrouve dans la fraction correspondant aux acides aminés

TABLEAU 1

Radioactivité spécifique des organes du rat après administration par os de $L-\left(U-{ }^{14} C\right) A P C-C a$ ou de $A P C-4{ }^{44} C a$ Activité spécifique

\begin{tabular}{|c|c|c|c|c|c|c|}
\hline \multirow{2}{*}{ Lots } & \multicolumn{2}{|c|}{ Rats adultes } & \multicolumn{3}{|c|}{ Rats ieunes } & \multirow{2}{*}{ Pro/Hyp } \\
\hline & $\left.3+4()^{1}\right)$ & $1+2\left(^{2}\right)$ & $\left({ }^{3}\right)$ & Pro $\left({ }^{4}\right)$ & Hyp ( $\left.{ }^{\mathbf{4}}\right)$ & \\
\hline 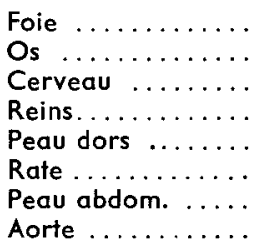 & $\begin{array}{r}55 \\
22 \\
5 \\
27 \\
11 \\
17 \\
19 \\
\end{array}$ & $\begin{array}{r}36 \\
1000 \\
20 \\
30 \\
17 \\
6 \\
18 \\
-\end{array}$ & $\begin{array}{r}1802 \\
5500 \\
400 \\
404 \\
214 \\
532 \\
155 \\
309\end{array}$ & $\begin{array}{l}\overline{20} \\
\overline{6} \\
\frac{6,2}{7,9} \\
-\end{array}$ & $\begin{array}{l}\overline{3,6} \\
\frac{-}{1,5} \\
\frac{-1}{2,1}\end{array}$ & $\begin{array}{l}3,8 \\
4,1\end{array}$ \\
\hline
\end{tabular}

(1) Lots ayant reçu $10 \mu \mathrm{Ci}$ de ${ }^{14} \mathrm{C}$-APC-Ca.

(2) Lots ayant reçu $10 \mu \mathrm{Ci}$ d'APC- ${ }^{45} \mathrm{Ca}$.

( $\left.{ }^{3}\right)$ Radioactivité spécifique (cpm/mg de tissu frais).

(4) Activité spécifique (cpm/ $\mu \mathrm{g}$ de proline ou d'hydroxyproline).
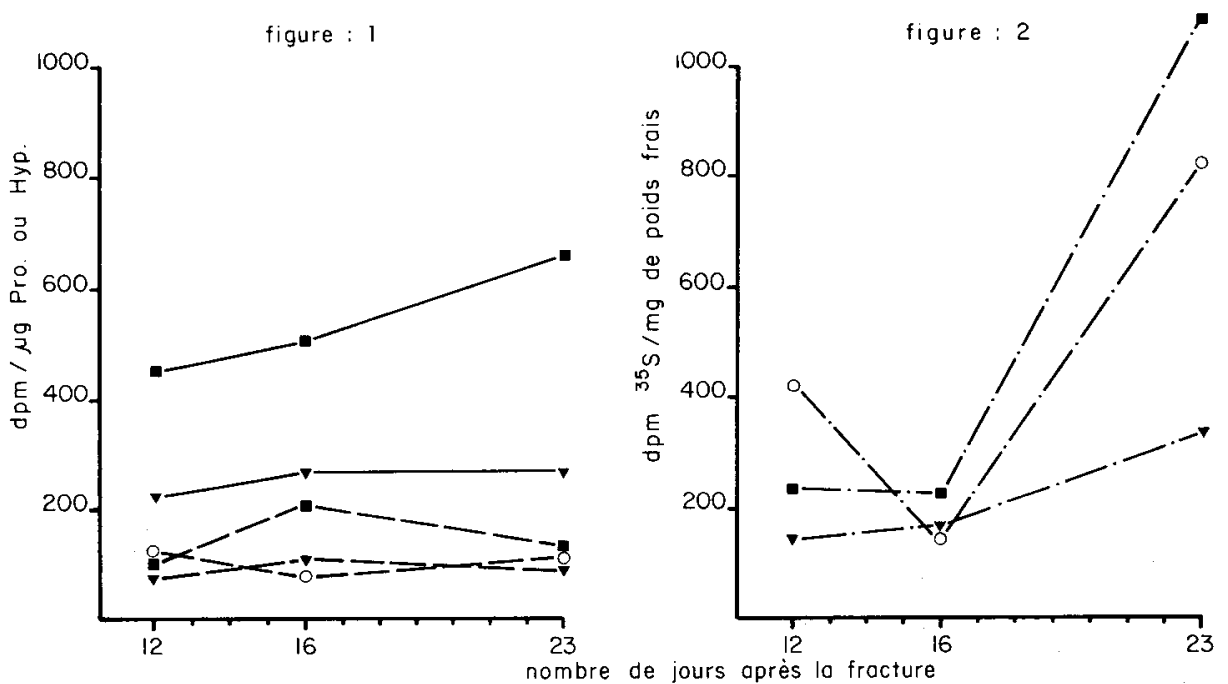

FIG. 1. - Activité spécifique de la proline — de l'hydroxyproline - - - des cals des rats $(300 \pm 15 \mathrm{~g})$ incubés avec la ${ }^{14} \mathrm{C}$-Proline.

$\Delta=$ témoins ; $0=100 \mathrm{mg} /$ jour d'APC-Ca ; $=300 \mathrm{mg} /$ jour d'APC-Ca.

FIG. 2. - Activité spécifique de ${ }^{35} \mathrm{~S}$ des cals des rats $(300 \pm 15 \mathrm{~g})$ incubés avec le $\mathrm{Na}_{2}{ }^{35 \mathrm{SO}_{4}}$. $\Delta=$ témoins ; o $=100 \mathrm{mg} /$ jour d'APC-Ca ; $=300 \mathrm{mg} /$ jour d'APC-Ca. 
neutres. Les acides glutamique et aspartique ainsi que la lysine ne sont que très faiblement radioactifs. L'activité spécifique élevée de la proline ef de l'hydroxyproline dans les « organes collagéniques » (os, peau) est en bon accord avec ce résultat. Le rapport élevé des activités spécifiques (AS) de la proline et de l'hydroxyproline (AS, Pro/A.S. Hyp) dans l'os suggère une stimulation sélective de la biosynthèse des protéines non collagéniques (Moczar ef al., 1977 ; Andrews et al., 1967 ; Rojkind et Gonzalez, 1974).

L'incorporation de proline dans les cals osseux de tibia du rat permet d'observer une accélération de la biosynthèse des protéines dès le début de la guérison (12 jours après la fracture) chez les animaux traités (fig. 1). La formation des glycosaminoglycannes sulfatés suivie par l'incorporation de ${ }^{35}$ S est également stimulée, particulièrement vers la fin de la guérison (fig. 2). L'activité spécifique plus élevée de l'hydroxyproline vers le $16^{e}$ jour de la guérison, permet de conclure à une accélération de la biosynthèse du collagène sous l'effet du traitement.

De cette étude, il ressort une incorporation rapide des métabolites de l'APC-Ca dans les tissus. La radioactivité liée à l'APC administré per os sous forme de sel calcique est très majoritairement retrouvée au niveau des acides aminés neutres.

D'autre part, le fait que la biosynthèse des glycosaminoglycannes et des protéines soit stimulée au cours de la formation de cals osseux chez le rat, indique que l'APC-Ca est susceptible de modifier la biosynthèse des macromolécules de la matrice osseuse.

\section{Réunion Groupe Développement INRA/Productions animales} Montpellier, 17-18 mai 1977.

\section{Références}

ANDREWS A. T., HERRING G., KENT P. W., 1967. Some studies on the composition of bovine cortical bone sialoprotein. Biochem. J., 104, 705-775.

BACHRA B. N., 1967. Some molecular aspects of tissue clacification. Clin. Orthop., 51, 199-222.

DI ROSA M., 1967. Antiammonemic and hepatoprotective action of a mixture of arginine, pyrrolidonecarboxylate, taurine and vitamin B6. Clin. terap., 42, 499-507.

LANGE W. E., CAREY E. E., 1966. Metabolism of ${ }^{14} \mathrm{C}$ labelled glutamic acid and pyrroglutamic acid animals. J. am. pharm. Ass., 55, 1147-1149.

MOCZAR E., BERENHOLC S., LAMENDIN H., ALLARD R., ROBERT A. M., 1977. Comparative study on the composition of the organic matrix of the long bones of the normal rat and of the mutant Op/Orl. Path. Biol., 25, 187-190.

MOCZAR E., ROBERT B., ROBERT L., 1972. Separation and determination of cross-linking aminoacids of elastin by high volfage paper electrophoresis. Analyt. Biochem., 45, 422-427.

PENTTINEN R., 1973. Metabolism of fracture callus of rat in vitro : II. Incorporation of ${ }^{35}$ S-sulphate, ${ }^{3} \mathrm{H}$-proline and ${ }^{32} \mathrm{P}$-phosphate. Acta physiol. scand., 87, 208-212.

RAMAKRISHNA M., KRISHNASWAMI P. R., RAJAGOPAL RAO D., 1970. Metabolism of pyrrolidonecarboxylic acid in rat. Biochem. J., 110, 895-897.

ROJKIND M., GONZALEZ E., 1974. An improved method for determining specific radioactivities of proline $-{ }^{14} \mathrm{C}$ and hydroxyproline $-{ }^{14} \mathrm{C}$ in collagen and in non collagenous proteins. Analyt. Biochem., 57, 1-7. 